\title{
Nuevos datos arqueológicos sobre el poblamiento altomedieval del Priorat (Tarragona)
}

\author{
New archaeological data on early medieval settlement of Priorat county \\ (Tarragona)
}

Marta Monjo*

Ignacio Montero

Núria Rafel ${ }^{\ddagger}$

\begin{abstract}
RESUMEN
Se presentan nuevos datos arqueológicos del nordeste peninsular (comarca del Priorat, Tarragona) relativos a un período poco conocido, el lapso temporal entre los siglos VII a X. Dichos datos han sido obtenidos en el curso de investigaciones arqueológicas cuyo objeto era la Prehistoria. La emergencia de informaciones relativas al período altomedieval en yacimientos prehistóricos incide en dos aspectos importantes. El primero de ellos es contribuir al estudio del poblamiento en este período en el que los datos parecen indicar la ausencia de una ruptura y de un vacío poblacional. El segundo se refiere a consideraciones metodológicas que ponen de manifiesto el sesgo que determinados prejuicios científicos han introducido en los datos.
\end{abstract}

Palabras clave: Período visigótico, Período altomedieval, Poblamiento rural, Palinología, Isótopos de plomo, Cataluña

\section{1.- INTRODUCCIÓN}

En el curso de las investigaciones llevadas a cabo en el marco de un proyecto centrado en la Prehistoria ${ }^{1}$ han aparecido algunos datos sobre el poblamiento altomedieval de la comarca del Priorat que, aunque limitados, suponen una aportación a un período histórico poco investigado en este territorio, y en general en el nordeste, y señalan algunos problemas metodológicos de la investigación

\begin{abstract}
New archaeological data from the Priorat County (Tarragona) are presented. They refer to a badly known period, the time span between the 7th to 10th centuries. These data have been obtained in the course of archaeological investigations whose object was Prehistory. The emergence of information related to the medieval period in prehistoric sites affects two important aspects. The first of them is to contribute to the study of the settlement within this period, in which the data seem to indicate the absence of rupture and population vacuum. The second refers to methodological considerations that highlight the bias that certain scientific prejudices have introduced into the data.
\end{abstract}

Keywords: Visigothic Period, Early Middle Age, Rural settlement, Palynology, Lead isotopes, Catalonia

llevada a cabo por los prehistoriadores e historiadores del siglo XX.

Se trata de datos que han sido recabados complementariamente a investigaciones centradas en la Prehistoria en dos localizaciones de una comarca, la del Priorat (Tarragona), que tradicionalmente se ha tratado como marginal y que se ubican en un espacio cronológico mal conocido (los siglos VI a X): la Coveta de l'Heura en Ulldemolins y la Mina

\footnotetext{
*Servei d'Arqueologia i Paleontologia, Departament de Cultura, Generalitat de Catalunya. mmonjog@gencat.cat. ORCID 0000-0003-2805-5647

† Instituto de Historia_CSIC. Ignacio.montero@cchs.csic.es. ORCID 0000-0003-0897-1031

‡ Arqueóloga.nrafel@historia.udl.cat. ORCID 0000-0001-9988-6080

1. El proyecto de referencia fue "Mineria i Metal-lúrgia a la Catalunya meridional: de la Prehistòria a Època Medieval". Las analíticas se financiaron con cargo al proyecto "Recursos minero-metálicos, intercambio y comercio en la Prehistoria y la protohistoria Peninsular (Cataluña y Norte del país Valenciano) HAR2014-54012-P.
} 
de la Turquesa en Cornudella de Montsant. Otros datos, más dudosos y difíciles de interpretar, proceden de un yacimiento situado ya en otra comarca, el Alt Camp, pero en el límite con la citada comarca del Priorat. Se trata de la Cova G, perteneciente al importante conjunto prehistórico del Cingle Blanc d'Arbolí (figura 1).

\section{2.- UN PERÍODO OSCURO}

El lapso temporal comprendido entre los siglos III y X, entre la crisis del mundo romano y las grandes madînas andalusíes, era poco conocido en Catalunya, aun cuando parecía probada la importancia del poblamiento rural. La investigación ha avanzado en las últimas décadas ofreciendo nuevos enfoques y nuevos estudios, aunque son diversas aún las lagunas por colmar, especialmente durante el lapso cronológico entre el fin del reino visigodo y el primer siglo de la ocupación islámica. La investigación reciente parece indicar que el territorio del noreste continuaba ocupado y en explotación. Quedan hoy completamente desfasadas las teorías que sostenían que los visigodos huyeron en masa hacia los Pirineos para protegerse de las huestes árabes (CLARAMUNT, 2014: 377). Por el contrario, la ruptura poblacional que se había propuesto parece cada vez más improbable. La entrada de los árabo-bereberes por el estrecho de Gibraltar no supuso la substitución de la población existente, habría sido necesaria la llegada de un inmenso contingente poblacional y, a su vez, la huida de la mayor parte de la población de Hispania; todo ello, por otra parte, se habría podido documentar arqueológicamente en los Pirineos o en Francia. Todo parece indicar, por el contrario, que la población permaneció y con ella sus costumbres, hábitats y cultura material (ZOZAYA, 2010: 236).

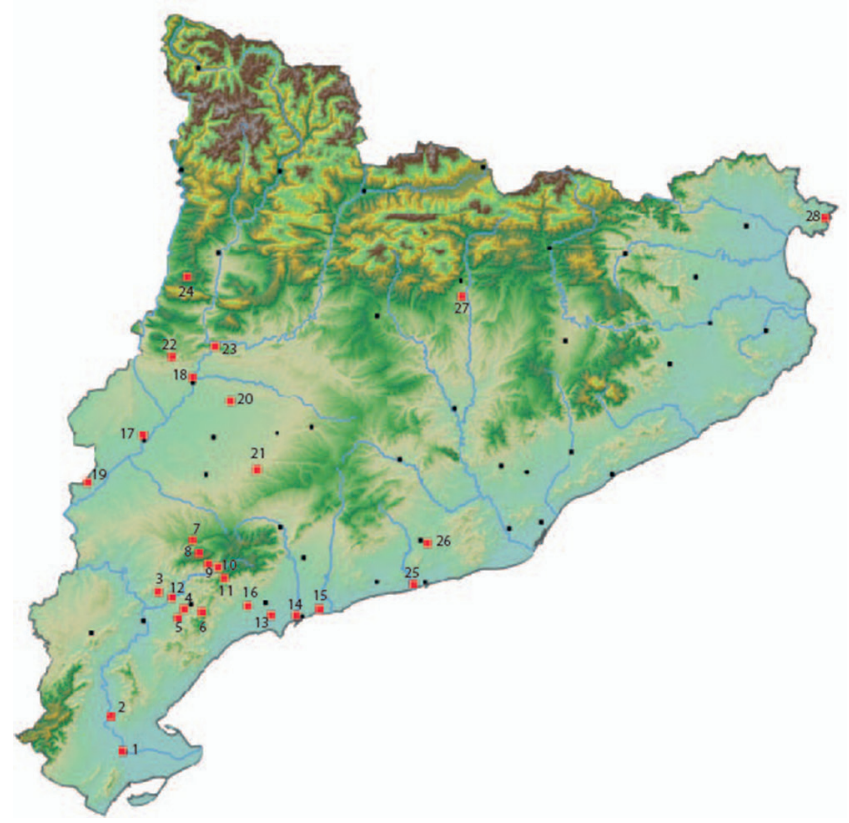

Fig. 1. Mapa de Cataluña con la localización de los yacimientos citados en el texto: 1. Amposta, 2. Tortosa, 3. Font del Molar, 4. Can Montagut (Marçà), 5. Serra de l'Espasa (Capçanes), 6. Torre de Fontaubella, 7. Coveta de l'Heura (Ulldemolins), 8. Albarca, 9. Mina de la Turquesa (Cornudella de Montsant), 10. Siurana, 11. Cova G (Arbolí), 12. Mina Regia (Bellmunt del Prio0rat), 13. Calipolis-Acequia Mayor (La Pineda), 14. Tarraco, 15. Els Munts (Altafulla), 16. L'Hort del Pelat (Riudoms), 17. Lleida, 18. Balaguer, 19. El Bovalar (Seròs), 20. Tossal del Moro (Castellserà), 21. Fogunussa (Sant Martí de Maldà), 22. Morulls (Os de Balaguer), 23. Palous (Camarasa), 24. Els Altimiris (Sant Esteve de la Sarga), 25. Cubelles, 26. Sant Miquel d'Olèrdola, 27. Serrat dels Tres Hereus (Casserres), 28. Port Lligat. 
No es hasta el último cuarto del siglo $X X$ cuando parece empezar un interés incipiente por el período visigodo en Catalunya. Hallazgos como el poblado y la basílica del Bovalar (Serós, Lleida) (PALOL, 1989), o Sant Miquel d'Olèrdola (Olèrdola, Barcelona) (ROVIRA et alii, 1991) incentivaron este interés. Actualmente son diversos los equipos que centran sus proyectos de investigación en este período y cada vez es mayor la luz que se aporta a su conocimiento. En esta línea se enmarcan los equipos de la Universitat de Barcelona encabezados, respectivamente, por Gisela Ripoll (RIPOLL, ARCE, 2001; MOLIST, RIPOLL, 2012; RIPOLL et alii, 2017) y Marta Sancho (SANCHO, ALEGRÍA, 2017; SANCHO, 2018), así como el liderado por Ramon Martí en la Universitat Autònoma de BarceIona (MARTí, 2013; FOLCH et alii, 2007; 2015) o las investigaciones del arqueólogo Joan Menchón para la zona de Tarragona (MENCHON, 2012; 20132)

Las dificultades de localización y de plena comprensión de los hábitats pueden explicar la falta de motivación para el estudio de este período histórico, que resultaba poco atractivo comparado con el esplendor y el legado arqueológico de la época romana. No se puede desestimar, por otra parte, la posibilidad de que el desconocimiento de la cultura material asociada y de los hábitats haya llevado a los arqueólogos, en alguna ocasión, a malinterpretar las evidencias. El desconocimiento y, por qué no, los prejuicios, pueden haber llevado a adscribir algunos materiales a un horizonte cultural y cronológico que no les correspondía. Además de los datos que más adelante presentamos sirva como ejemplo el pequeño yacimiento del Tossal del Moro (Castellserà, Lleida). Tradicionalmente se había considerado un yacimiento ibérico y las diferentes excavaciones aparentemente lo habían corroborado; ahora bien, la localización, en el interior de unos silos, de unas hebillas visigodas y unos cardadores de lana datados en el siglo VIII, hizo replantear algunas interpretaciones asumidas con anterioridad. Los visigodos habían aprovechado el urbanismo del antiguo poblado íbero, reocupándolo con pocas modificaciones (ESCALA et alii, 2011; 2018). El del Tossal del Moro de Castellserà no es un caso único, un ejemplo similar puede encontrarse en el Serrat dels Tres Hereus (Casserres, Barcelona) (FOLCH et alii, 2007) que la historiografía tradicional había interpretado como un yacimiento exclusivamente ibérico. Ello sugiere que habría que plantear el reestudio de algunos materiales y yacimientos, y cuestionar algunas de las premisas de las que todos hemos partido.

En el caso concreto del Priorat, que aquí nos ocupa, el vacío parece ser aún mayor. Su orografía compleja le otorga la etiqueta de tierra agreste y marginal, imagen que se viene arrastrando desde la Edad Media, cuando se la incluye entre las vastae solitudines en los textos posteriores a la conquista del siglo XII (MENCHON, 2013: 61). Sin embargo, la visión de los cronistas árabes es muy distinta. Lejos de considerar estas tierras desérticas y baldías, sus crónicas hablan de tierras fértiles, ricas y explotadas. Al-Bakri reporta que eran muy conocidas las minas de galena del distrito de Tortosa (BRAMON, 2000: 53, 87; RAFEL, ARMADA, 2010: 256), mientras que al-Zuhrī, a su vez, destaca la explotación de las abejas (BRAMON, 1998: 71). El estado actual de la investigación sitúa el Priorat de los siglos X-XII dentro del distrito de la madîna de Tortosa, en un territorio conocido como al-Barka, al frente del cual se encontraba la fortaleza de Siurana, desde la que se comandaba el territorio y se guardaba la vecina frontera. La población se repartía en pequeños núcleos ganaderos y almunias como la de Albarca o la de la Torre de Fontaubella (BOLÓs et alii, 2016: 58, 61). Las excavaciones arqueológicas llevadas a cabo en el castillo de Siurana (PIERA, MENCHON, 2011) no han contribuido hasta ahora a mejorar la percepción obtenida a través de la documentación escrita. Las grandes intervenciones arqueológicas se han llevado a cabo en Tortosa, Lleida o Balaguer aportando

2. Agradecemos a Joan Menchon sus comentarios al texto. Por supuesto, cualquier carencia en el mismo es debida exclusivamente a los firmantes. 
información sobre las ciudades, pero nada, o poco, sobre la vida rural. Y es que conocemos arqueológicamente las grandes madînas catalanas de los siglos X-XII, pero poco del mundo rural que las circundaba y menos de las mismas ciudades y su territorio antes del siglo $X$ (ALÒS et alii, 2007).

Almunias, alquerías, necrópolis y mezquitas rurales eran, y son, las grandes desconocidas en la arqueología catalana, motivo por el cual la descripción de estos territorios como lugares sin cultivar, habitados por gentes casi salvajes encajaba muy bien con el estado de la investigación arqueológica del territorio. Así pues, entre la desaparición del imperio romano y la implantación del califato de Córdoba existía un aparente vacío, que en realidad ha resultado no serlo.

\section{3.- LOS NUEVOS DATOS Y SU CONTEXTUALIZACIÓN}

3.1.- La Coveta de l'Heura (Ulldemolins): es una cavidad paradolménica de larga ocupación que tuvo diversas funciones a lo largo de su período de uso (VILASECA, 1952; RAFEL et alii, 2016). En un primer momento la cavidad se utilizó como taller para la fabricación de hojas de sílex foliáceas y como lugar de habitación. Pero ya a mediados del III milenio cal BC se inició una nueva fase en la que se modificó la cavidad y se utilizó como lugar de enterramiento colectivo. Fragmentos de mineral y gotas de fundición atestiguan que el yacimiento tuvo también un uso como taller metalúrgico; no obstante, no ha sido posible determinar si este uso corresponde a la fase Calcolítica o hay que situarlo ya en el Bronce Antiguo-Medio. Se realizaron tres dataciones radiométricas sobre hueso humano con la finalidad de fechar la fase de enterramientos que el descubridor del yacimiento, Salvador Vilaseca, había considerado como íntegramente prehistórica. Sin embargo, dos de las dataciones realizadas corresponden a época altomedieval (siglos VII a X cal AD), mientras que solo la tercera se sitúa en la Prehistoria, a mediados del III milenio (2618-2491 cal BC) (RAFEL et alii, 2016: 112). Esta última es coherente con el hecho de que la mayor parte del importante lote de materiales recuperados en el yacimiento es encuadrable tipológicamente en el Calcolítico Reciente. Las dos primeras tienen la virtud de poner de manifiesto que, aunque el osario de la cueva tuvo una fase Calcolítica, también tuvo una fase de enterramiento en época medieval. Una de las fechas (Beta 299206) se sitúa entre 612 y 651 cal AD [a 1 s y al $100 \%$ de probabilidad], mientras que la otra (Beta 299207) da una datación de 771-903 cal AD [a 2 s y al $84,8 \%$ de probabilidad] o de 918-964 cal AD [a 2 s y al 15,1\% de probabilidad] $]^{3}$.

3.2.- Mina de la Turquesa (Cornudella de Montsant): Entre 2012 y 2015 se llevaron a cabo excavaciones en la Mina de la Turquesa (conocida también como Mina del Mas de les Moreres), donde había sido hallado un pico de minero prehistórico en prospección. En el curso de estas investigaciones se localizó un pozo minero (L1) cuya tecnología de excavación apuntaba a una cronología preindustrial. Dadas las dificultades inherentes a la datación de minas se optó por fechar radiocarbónicamente el polen recuperado en los niveles de relleno de dicha labor, así como una muestra de sedimento del mismo relleno. La primera (Beta 434530) proporcionó una datación convencional de 1260+30 BP (689-751 cal AD [85\%] a 1s y $669-778 \mathrm{cal} A D[89,7 \%]$ a 2 s) y la segunda (Beta 423141) de 1110+30 BP (895-928 cal AD [48,6\%] y 940-976 cal AD [51,3\%] calibrada a 1 s y $879-1013$ cal BC $[100 \%]$ a $2 s^{4}$. Es decir, las dataciones nos sitúan la obliteración y relleno definitivo del pozo en un lapso de tiempo entre los siglos VII y X (HUNT, RAFEL y SORIANO 2018: 16-18) y la muestra de polen entre la segunda mitad del siglo VII y los tres primeros cuartos del siguiente.

3. Curva Intcal 13.14c, Calib 7.0.0 Stuiver and Reimer 1986-2013 (REIMER et alii, 2013).

4. Calib Rev. 7.1, curva de calibración empleada IntCal13 (REIMER et alii, 2013). 
El análisis palinológico (PÉREZ DÍAZy y LÓPEZ SÁEZ, 2018) reveló un paisaje vegetal dominado por un cortejo de tipo mediterráneo, con una cierta influencia submediterránea y eurosiberiana. El estudio documentó evidencias claras de deforestación, de antropización y de cultivo de cereales en las proximidades, así como la presencia de Olea.

En el entorno inmediato a la Mina de la Turquesa no existen por el momento otros paralelos con los que poder comparar estos datos, convirtiendo este estudio en el único disponible hasta la fecha en la zona que refleje la vegetación en la comarca del Priorat durante los siglos VII y VIII. Sin embargo, resulta interesante poner estos datos en relación con los obtenidos en la zona 4 de la columna polínica de la Acequia Mayor de la Pineda (Vilaseca), localidad situada en la costa de Tarragona, a $50 \mathrm{~km}$ de la Mina Turquesa. Esta columna fue extraída en agosto de 2007, alcanzando una profundidad máxima de $277 \mathrm{~cm}$. La zona 4, situada entre 110 y $95 \mathrm{~cm}$ de profundidad, se dató del 600 al 1050 d.C. y se corresponde con una fase de deforestación y, aquí también, con una reducción de los valores de polen arbóreo (RIERA et alii, 2010: 169). En ambos casos se documenta Olea, que en la Acequia se interpreta como un taxón cultivado, y cereales. La deforestación entre los siglos VII y VIII se ha evidenciado también en Cubelles (BarceIona) (RIERA, 2003) y en Amposta (Tarragona) (FOLLIERI et alii, 2000). Los resultados de Amposta y la acequia de la Pineda se han vinculado a una extensión del cultivo de cereales y olivos, si bien en Amposta la ganadería podría también estar vinculada a estos cambios, como sucede en Cubelles (RIERA et alii, 2010: 171). Estudios palinológicos de cronología similar se localizan más al norte, en el Ampurdán, donde desde el siglo VIII cal AD se documenta una intensa deforestación de los bosques costeros. El depósito de Portlligat (cabo de Creus) refleja a su vez características similares, en este caso un bosque abierto con evidencias de ganadería y cultivo (PÉREZ DÍAZ y LÓPEZ SÁEZ, 2018: 45).
3.3.- Cova G de Arboli: No podemos dejar de reflejar en estas líneas la revisión de otro hallazgo de Vilaseca de difícil interpretación, pero que, de nuevo, incide claramente en el problema de una sobreinterpretación de la Prehistoria en los yacimientos de la comarca prioratina y su entorno. Se trata de unos fragmentos de metal exhumados en el gran conjunto rupestre prehistórico del Cingle Blanc (Arbolí) que VILASECA (1934) analizó y, al constatar que se trataba de restos de piezas de cobre puro, interpretó como restos atribuibles a fragmentos de puñales y cuchillos prehistóricos. Se trata de una serie de fragmentos informes (15 en total), que proceden de planchas cortadas a cincel, algunos de los cuales conservan remaches o los orificios para los mismos (FIGURA 2). Fueron hallados en la Cova $\mathrm{G}$ del conjunto mencionado. Dicha cueva proporcionó escasos restos consistentes en un punzón de hueso y diversos fragmentos de cerámica prehistórica elaborada a mano, todos lisos a excepción de uno de ellos que presentaba una decoración de incisiones. Tres de los fragmentos metálicos fueron analizados en el proyecto Arqueometalurgia de la Península Ibérica (ROVIRA, MONTERO, CONSUEGRA, 1997: 363) y valorados también como de un momento avanzado de la Edad del Bronce (Bronce Medio) dado que formalmente no encajaban en un momento Calcolítico, pero la composición no permitía descartar cronologías prehistóricas. Se han realizado nuevos análisis de composición de cinco de los fragmentos (figura 2, números 1 a 5, y Tabla 1) y de isótopos de plomo de tres de ellos (FIGURA 2, números 3, 4 y 5, y Tabla 2). El estudio de estos fragmentos ha mostrado que, aunque se trata de cobre sin alear, la forma y tipología de las piezas a las que pertenecieron no se corresponden con época Calcolítica o de la Edad del Bronce. El análisis de isótopos de plomo muestra una posible procedencia que no corresponde a la Península Ibérica ni a las zonas del entorno mediterráneo. La edad geológica calculada a partir de los isótopos señala a formaciones de gran antigüedad. En dos de ellos entre 1500 y 1600 millones de años, y el tercero de 630 millones de años. 


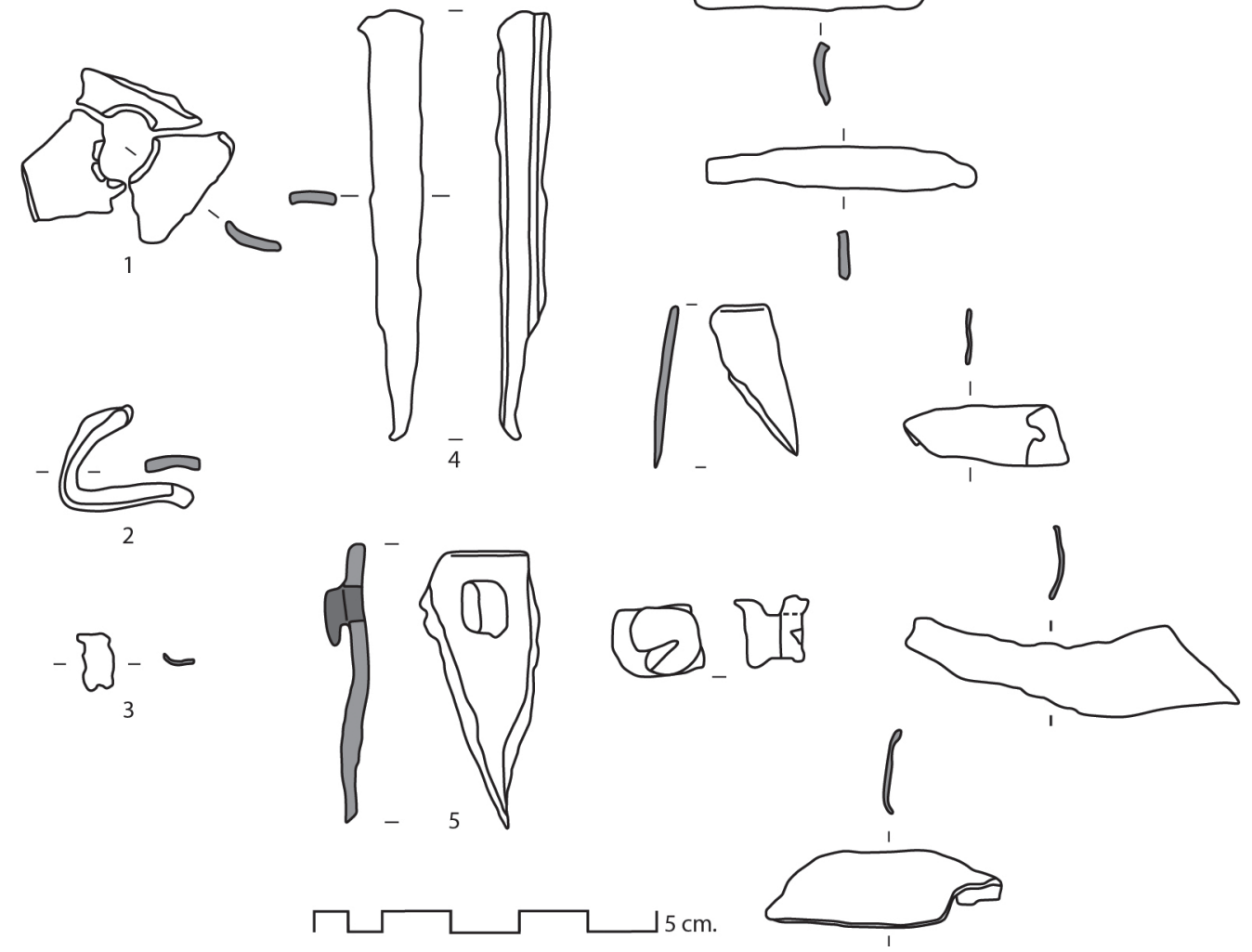

Fig. 2. Fragmentos de cobre de la Cova G (Arbolí). Numerados del I al 5 los que han sido objeto de análisis de composición. Los números 3,4 y 5 corresponden a los sometidos a analítica de isótopos de plomo.

Tabla 1. Cova G. Análisis elemental mediante pXRF utilizando el espectrómetro INNOV-X del Museo Arqueológico Nacional. Valores expresados en \% en peso. Sobre límites de detección, condiciones de análisis y preparación de muestra consultar ROVIRA LLORENS y MONTERO RUIZ (2018).

\begin{tabular}{|l|l|c|c|c|c|c|c|c|c|c|c|c|}
\hline ANÁLISIS & TIPO & $\mathbf{N}^{\mathbf{0}}$ & $\mathbf{F e}$ & $\mathbf{N i}$ & $\mathbf{C u}$ & $\mathbf{Z n}$ & $\mathbf{A s}$ & $\mathbf{A g}$ & $\mathbf{S n}$ & $\mathbf{S b}$ & $\mathbf{P b}$ & $\mathbf{B i}$ \\
\hline PA24790 & Remache & 5 & $<0.02$ & 0,06 & 99,0 & $<0.1$ & 0,14 & 0,19 & $<0.02$ & $<0,15$ & 0,63 & $<0.05$ \\
\hline PA24791 & $\begin{array}{l}\text { Recorte } \\
\text { fino }\end{array}$ & 3 & $<0.02$ & 0,1 & 98,7 & $<0.1$ & 0,17 & 0,21 & $<0.02$ & $<0.15$ & 0,8 & $<0.05$ \\
\hline PA24792 & $\begin{array}{l}\text { Recorte } \\
\text { grueso }\end{array}$ & 4 & $<0.02$ & 0,05 & 98,7 & $<0.1$ & 0,79 & $<0,15$ & 0,07 & $<0.15$ & 0,35 & $<0.05$ \\
\hline PA24793 & $\begin{array}{l}\text { Recorte } \\
\text { grueso } \\
\text { doblado }\end{array}$ & 2 & $<0.02$ & 0,06 & 98,8 & $<0.1$ & 0,69 & $<0.15$ & $<0.02$ & $<0,15$ & 0,42 & $<0.05$ \\
\hline PA24794 & $\begin{array}{l}\text { Recorte } \\
\text { fino } \\
\text { agujero }\end{array}$ & 1 & 0,09 & 0,09 & 99,2 & $<0.1$ & 0,38 & $<0.15$ & 0,06 & $<0.15$ & 0,22 & $<0.05$ \\
\hline
\end{tabular}

Depósitos de antigüedad superior a los 1000 millones de años solo se dan en Europa en los países escandinavos. Mineralizaciones de cobre de esta edad (1,7-0,9 mA) aparecen en el escudo Báltico o Fenoscandio en la provincia
Gneis suroccidental, siendo aún más antiguas las de la provincia Svecofeniana, donde se sitúan las minas de Falun cuyos valores isotópicos podrían tener relación con los cobres de la Cova G. Estas minas de Falun abastecieron 
Tabla 2. Cova G. Análisis de isotopos de plomo realizados con la técnica MC-ICP-MS en los laboratorios de Geocronología (SGIker) de la Universidad del País Vasco.

\begin{tabular}{|l|l|c|c|c|c|c|}
\hline ANÁLISIS & \multicolumn{1}{|c|}{ OBJETO } & $\mathbf{2 0 8 / 2 0 6}$ & $\mathbf{2 0 7 / 2 0 6}$ & $\mathbf{2 0 6 / 2 0 4}$ & $\mathbf{2 0 7 / 2 0 4}$ & $\mathbf{2 0 8 / 2 0 4}$ \\
\hline PA24790 & Remache & $\mathbf{2 , 2 0 8 4 2}$ & 0,94770 & $\mathbf{1 6 , 2 6 8 5}$ & 15,4177 & 35,9278 \\
\hline PA24791 & Recorte fino & 2,21950 & 0,95740 & 16,0839 & 15,3992 & 35,6975 \\
\hline PA24792 & $\begin{array}{l}\text { Recorte } \\
\text { gruesO }\end{array}$ & $\mathbf{2 , 1 2 0 7 6}$ & $\mathbf{0 , 8 7 6 5 3}$ & $\mathbf{1 7 , 7 7 2 0}$ & $\mathbf{1 5 , 5 7 7 7}$ & $\mathbf{3 7 , 6 9 0 3}$ \\
\hline
\end{tabular}

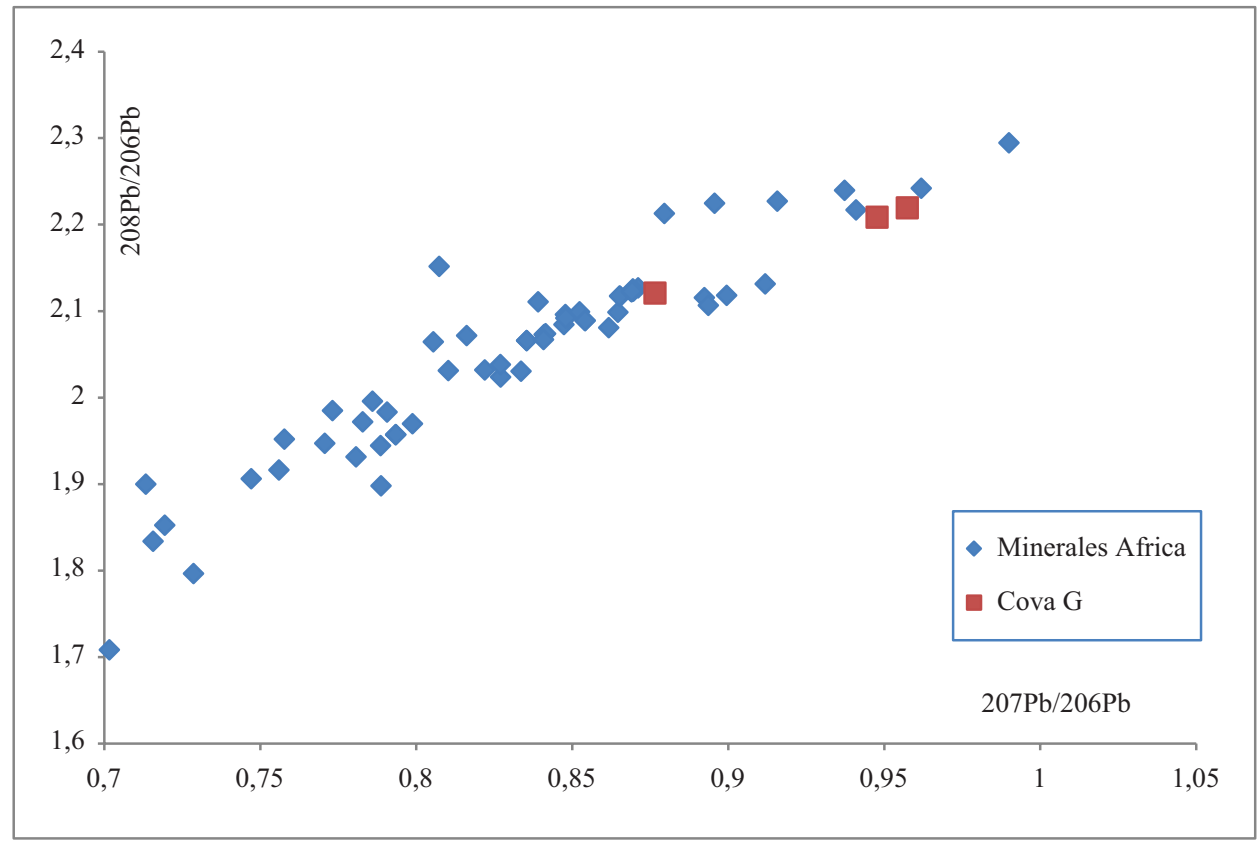

Fig. 3. Representación de los análisis de isótopos de plomo de la Cova G (Tabla 2) en relación a los datos disponibles de minerales de cobre africanos.

a gran parte de Europa, incluidos los reinos hispánicos, entre los siglos XVI y XVIII. No hay referencia de explotación de estas minas en la Prehistoria (LING et alii, 2013) (figura 3).

La otra opción geográfica para mineralizaciones de tanta antigüedad es África. Los datos geológicos que hemos podido manejar indican que los depósitos de Rooiberg (Sudáfrica), aunque también son algo más antiguos genéticamente, tienen signaturas isotópicas próximas a los lingotes de la Cova G (figura 4). La posibilidad de llegada de cobre de esta zona africana a la península solo tiene sentido en la ruta comercial portuguesa hacia la India, establecida a partir del siglo XV. Si bien también deberíamos considerar la opción del comercio medieval islámico con el África oriental con monedas islámicas conocidas en el área del Gran Zimbawe desde donde se comerciaba con oro a través de la ruta del mar Rojo (MERI 2006: 816818). Sin embargo, no se han identificado en los análisis de isótopos de plomo de lingotes de cobre del Centro y Sur de África las signaturas isotópicas de las minas de Rooiberg (RADEMAKERS et alii, 2019), pero sí ajustan con las minas del cinturón Damaran-Lufilian con el que el tercer fragmento de la Cova G también podría coincidir. Si bien la signatura isotópica en este caso podría tener relación con algunas minas de Sierra Morena occidental, la aparición conjunta con los otros fragmentos y su similitud tipológica deben hacernos buscar una única interpretación para todos ellos. 


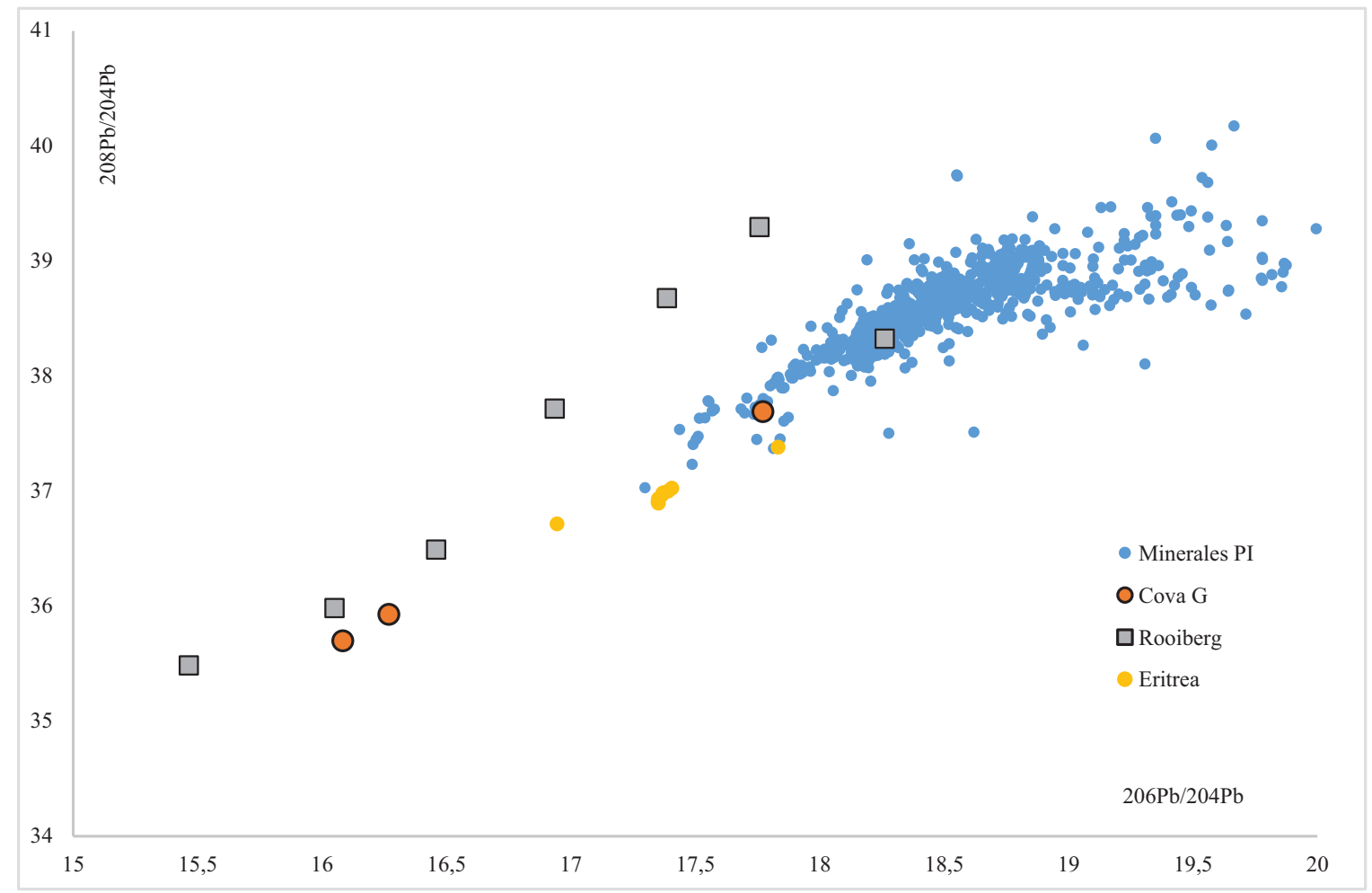

Fig. 4. Representación de los isótopos de plomo de los metales de la Cova G (Tabla 2) en relación a los minerales de la Península Ibérica (PI) y los minerales africanos de Rooiberg (Sudáfrica) y Eritrea.

Aunque no tenemos una interpretación satisfactoria concreta para explicar estos fragmentos de cobre, los datos señalan que no son prehistóricos y que, a partir del período medieval o en época moderna, pudieron haber llegado desde el Norte de Europa o del Sur de África. También se nos escapan los motivos de su ocultamiento fragmentados en la Cova G.

\section{4.- DISCUSIÓN DE LOS DATOS}

Los hábitats que sin duda debían asociarse directamente a estas evidencias de enterramientos y de deforestación y cultivo nos son por ahora desconocidos, aunque algunos datos permiten formular hipótesis sobre su carácter.

Sí conocemos hallazgos cronológicamente anteriores, de época romana, como las villas de Can Montagut (Marçà) (JÁRREGA, 2017) y la Font del Molar (ARMADA et alii, 2010), un posible horno cerámico en la Serra de l'Espasa de
Capçanes (MENCHON, 2013: 59) y las lucernas romanas de la mina Blancardera en Bellmunt del Priorat (RAFEL, ARMADA, 2010: 249-250). Estas últimas permiten sugerir que en época romana se benefició el plomo de la comarca, para el que se ha propuesto su identidad con el plumbum nigrum oleastrense mencionado por Plinio (RAFEL, ARMADA, 2010). Hay que tener en cuenta, por otra parte, que a partir al menos del siglo XI la galena (alcohol) de esta cuenca minera era conocida por su calidad y era exportada a Oriente (VALLVÉ, 1980; 1996; BRAMON, 2000: 87, § 53).

La laguna de información en relación al poblamiento altomedieval se debe en buena parte a que las villas romanas, aunque modificadas, podrían haber pervivido hasta el siglo X como lugar de hábitat en las zonas del Camp de Tarragona, la Conca de Barberà y el Priorat (MENCHON, 2012). Sin embargo, otros autores abogan por un abandono de las villas y la aparición de nuevos asentamientos muy cercanos a estas (FOLCH et alii, 2015: 98). Ambas 
teorías no están disociadas; resulta innegable que muchas villas se abandonan, pero el hecho de construir nuevos hábitats muy cercanos a estas, o incluso aprovechando las partes rusticae, muestra una voluntad de continuidad en la zona de hábitat como se ha documentado en el yacimiento de Vilauba (Banyoles, Girona) (CASTANYER et alii, 2012). La reutilización de estos espacios no responde tanto a la voluntad de aprovechar los antiguos edificios sino a la continuación de la explotación del espacio agrícola vinculado a las antiguas uillae (BROGIOLO, CHAVARRIA, 2008: 204).

En época romana el Priorat se encontraba dentro del ager tarraconensis y, por tanto, en el área de influencia de la capital (MENCHON, 2013). A lo largo del Bajo Imperio, se observa un cambio en el patrón de asentamiento, las ciudades dejan de jugar un papel clave frente al campo y es que parece producirse un proceso de ruralización de la población, proceso que se ha documentado de forma generalizada, con variantes geográficas, en todo el Imperio (BROGIOLO, CHAVARRIA, 2008). Así pues, Tarraco pierde peso urbano y la población se ruraliza. Este hecho no significa necesariamente que las ciudades se abandonen. En Tarragona se han documentado reformas en el puerto, se construye un horreum en el siglo VII y dos más en el siglo VIII, cuando se realiza una importante reforma urbana para la construcción de espacios industriales (DÍAZ et alii, 2015).

No se ha contrastado fehacientemente en el Priorat la continuidad de estas villas que podrían perdurar durante la tardoantigüedad y la alta Edad Media, aunque las necrópolis asociadas a ellas, como la de la Font (El Molar) (VILASECA, 1930; ARMADA et alii, 2010) - Can Montagut (Marçà) (MENCHON, 2012: 143; JÁRREGA, 2017) podrían vincularse a este momento. Con la voluntad de mostrar la continuidad en la ocupación del territorio algunos autores ponen en relación la necrópolis altomedieval del Mas de Palau con la almunia de Albarca, obteniendo de esta manera una secuencia continuada de ocupación desde la tardoantigüedad. Con el mismo objetivo, se relaciona la almunia de la Torre de Fontaubella con un camino de origen romano (BOLÓS et alii, 2016: 61).

En el territorio circundante, aún dentro del ager tarraconensis, se han localizado algunas villas que se ocupan más allá del siglo V, no ya como villas stricto sensu, sino adaptadas y modificadas de forma substancial. Su perduración se conoce a través de las reformas arquitectónicas y amortizaciones documentadas, las inhumaciones asociadas a estos niveles de reforma y algunos materiales, aunque mayoritariamente la cultura material parece tener una continuidad. Este es el caso de la villa de Cal-lípolis (Vilaseca, Tarragona) (DÍAZ, MACÍAS, 2008) o las inhumaciones de la villa de els Munts (Altafulla, Tarragona) ambas datadas entre los siglos III y IV d.C. aunque la necrópolis podría perdurar hasta los siglos VI-VII d.C. (GARCIA et alii, 1999); o la inhumación en decúbito lateral de la villa de l'Hort del Pelat (Riudoms, Tarragona) ya claramente de ritual islámico (AROLA, BEA, 2004). Todas estas villas presentan reformas o amortizaciones y ocupaciones en época tardía, a partir de los siglos III-IV d.C., que transforman los antiguos espacios de hábitat o los espacios termales para nuevos usos agrícolas o productivos e incluso en necrópolis, fenómeno que puede extrapolarse a gran parte de los territorios occidentales del Imperio (RIPOLL, ARCE, 2001).

La población ocupó el campo en hábitats tipológicamente diversos: reocupando antiguas villas o en nuevos emplazamientos muy próximos a estas, aprovechando estructuras anteriores, como en el Tossal del Moro (ESCALA et alii, 2011; 2018) o el Serrat dels Tres Hereus (Casserres, Barcelona) (FOLCH, et alii, 2007), en conjuntos de cabañas como els Altimiris (Sant Esteve de la Sarga, Lleida), un asentamiento creado ex novo (SANCHO, 2018; SANCHO, ALEGRÍA, 2017), o basílicas asociadas a poblados o centros monásticos, como Bovalar (Seros, Lleida) (PALOL, 1989; SALES, 2014). Se aplicaron nuevas estrategias productivas que hay que asociar a la deforestación documentada, que a su vez puede vincularse con un auge del 
pastoreo $^{5}$, si bien en el caso del Priorat y la mina de la Turquesa puede estar relacionado con la propia actividad minera.

Este sería el modelo de ocupación que perduraría, con modificaciones paulatinas, hasta finales del siglo IX y principios del $X$ en la actual zona de Catalunya. La entrada de los árabo-bereberes por el estrecho de Gibraltar no supuso la expulsión en masa de la población indígena. Se cambió el régimen de gobierno y el modelo de explotación del territorio y paulatinamente se produjo el proceso de aculturación, pero no una ruptura, hecho que no implica que no hubiese lucha contra el invasor y violencia. A nivel hispánico, quizás uno de los ejemplos de continuidad mejor conocido es el Tolmo de Minateda (Hellín, Albacete) (GUTIÉRREZ 2007, 2013). En Catalunya no se ha encontrado un yacimiento en el que se haya podido documentar esta transición, si bien excavaciones recientes como la realizada en el antiguo hospital de clérigos de Girona, donde se documentaron ocho inhumaciones que seguían el ritual islámico, datadas en el siglo VIII y que compartían el espacio funerario con otras sepulturas cristianas, aportarán nuevos datos para la comprensión de este período de transición. Resulta especialmente relevante el hecho de que mediante análisis de ADN se ha podido determinar que de las ocho inhumaciones que seguían el ritual islámico una pertenecía a un individuo procedente de la zona del Golfo Pérsico y otra a un individuo del Magreb (FUENTES, en prensa) mientras que el resto pertenece a población indígena.

El mantenimiento de la población explicaría la pervivencia y adaptación de los lugares de hábitat, explotación, sepultura y culto y, a su vez, justificaría la dificultad de interpretación de la cultura material, en la que no se produciría una ruptura clara. Gradualmente se va produciendo una islamización hasta la implantación del Califato de Córdoba, cuando el nuevo estado promoverá la aparición de nuevos asentamientos rurales y llevará a las ciudades a convertirse en las madînas que conocemos, pero será ya a partir del siglo X.

Sin más datos directos sobre yacimientos del Priorat comprendidos entre los siglos $V$ y $X$, no se puede dotar de un mayor contexto poblacional a la mina de la Turquesa, a las inhumaciones de la Coveta de l'Heura y a la deforestación documentada a través de los análisis polínicos. Resulta, por el momento, imposible asociar a unas comunidades concretas la explotación de estos recursos y, si bien se puede hablar de continuidad en la explotación minera prácticamente desde la prehistoria (RAFEL et alii, 2018; SORIANO, HUNT, 2018; RAFEL et alii, 2016; MARTÍNEZ ELCACHO, 2014), cabe pensar que los hábitats de estos explotadores se situaban en la zona circundante. La investigación arqueológica de esta comarca aportará, sin duda, nuevos datos para el conocimiento de estos hábitats.

Todo lo expuesto hasta aquí indica claramente que la obscuridad del período que aquí tratamos se enraíza, más que en una falta de poblamiento, en el carácter de este, que lo hace poco visible arqueológicamente hablando y, por otra parte, en carencias claras de la investigación -geográficamente muy desigual- llevada a cabo hasta la fecha, que con las líneas precedentes queremos poner en evidencia con el objetivo de que futuras investigaciones huyan de los prejuicios de que han adolecido las llevadas a cabo hasta ahora.

\section{BIBLIOGRAFÍA}

ALÒS, Carme; CAMATS, Anna; MONJO, Marta; SOLANES, Eva (2007): "Organización territorial y poblamiento rural en torno a Madîna Balagí (siglos VIII-XII). En SÉNAC, Philippe (éd.), Villes et campagnes de Tarraconaise et d'al-Andalus (Vle-Xle siècles): la transition, pp.

\footnotetext{
5. El hallazgo de un hierro para pelar pieles en una de las inhumaciones de la necrópolis de Palous muestra la relevancia del ganado y la piel (SOLANES, ALÒS, 2003). En el momento de redactar el articulo las autoras situaron la necrópolis, por paralelos, en el siglo VII-VIII. Dataciones de C ${ }^{14}$ realizadas en el año 2013 por el Laboratori de Datació per Radiocarboni de la Universitat de Barcelona por encargo el Servei d'Arqueologia i Paleontologia de la Generalitat de Catalunya; del individuo PA99/ent.4/UE10 arrojaron una datación cal DC 432-594.
} 
157-181. Paris: CNRS; Toulouse: Université de Toulouse-Le Mirail. https://doi.org/10.4000/books.pumi.25693

ARMADA, Xóse-Lois; GRAELLS, Raimon; RAFEL, Núria; PAYÀ, Xavier (2010): "La presencia romana en la Font del Molar (Priorat, Tarragona): prospección de superficie y hallazgos fortuitos". Revista d'Arqueologia de Ponent 20, pp. 177-190.

AROLA, Roc; BEA, David (2004), "La vil.la romana de l'Hort del Pelat (Riudoms, Baix Camp)". Butlletí Arqueològic 24, pp 75-95.

BOLÓS, Jordi; BONALES; Jacinto, FLÓREZ, Marta; MARTíNEZ ELCACHO, Albert (2016): Caracterització histórica del paisatge del Priorat-Montsant-Siurana. Recuperado de: http://www.catpaisatge. net/pahiscat/docs/CHPC_PRIORAT-MONTSANT-SIURANA.pdf

BRAMON, Dolors (1998): Nous textos d'historiadors musulmans referents a la Catalunya medieval (continuació de l'obra de J. Mª Millàs i Vallicrosa) (tesis doctoral). Universitat de Barcelona. Barcelona. Recuperado de: http://hdl.handle.net/10803/2071

BRAMON, Dolors (2000): De quan érem o no musulmans: textos del 713 al 1010: continuación de l'obra de J.M. Millàs i Vallicrosa. Barcelona: Eumo

BROGIOLO, Gian Pietro; CHAVARRÍA, Alexandra (2008): "El final de las villas y las transformaciones del territorio rural en el Occidente (siglos V-VIII)". En FERNÁNDEZ OCHOA, Carmen, GARCÍA-ENTERO, Virginia y GIL SENDINO, Fernando (eds.), Las villae tardorromanas en el occidente del Imperio: arquitectura y función. IV Coloquio Internacional de Arqueología en Gijón, pp. 193-213. Gijón: TREA.

CASTANYER, Pere, TREMOLEDA, Joaquim y DEHESA, Rafel (2012): "De Vilauba a Villa Alba. L'hàbitat dels segles VI-VII d.C. de la vil.la romana de Vilauba (Camós, Pla de l'Estany)". Tribuna d'Arqueologia 2010-2011, pp. 9-21. Barcelona: Generalitat de Catalunya.

CLARAMUNT, Salvador (2014): "La formación de Cataluña y su inserción en la Edad Media española”. En CLARAMUNT, Salvador Societat, cultura i món mediterrani. Recull d'articles. Collecció Homenatges, 47, pp. 375-392. Barcelona: Universitat de Barcelona.

DÍAZ, Moisés; GIMENO, Marc; MESAS, Imma (2015): "Nuevos datos sobre la evolución del área portuaria occidental y fluvial de Tarraco. Últimas excavaciones en la UA 15 y en la c/ Vidal i Barraquer (Antigua Sofrera Pallarès)". En LÓPEZ VILLAR, Jordi (ed.), Tarraco Biennal. Actes del 2on. Congrés Internacional d'Arqueologia i Món Antic. August i les províncies occidentals; 2000 aniversari de la mort d'August. Vol. 2, pp. 229-236.Tarragona: Fundació Privada Mútua Catalana.

DÍAZ, Moisés; MACIAS, Josep Maria (2008): "La vil-la romana de la Pineda/Cal'lípolis (Vila-seca, Tarragonès)". En REMOLÀ, Josep Anton (coord.), El territorio de Tarraco: vil.les romanes del Camp de Tarragona. Actes del Seminari organitzat pel Museu Nacional Arqueológic de Tarragona, la Societat Catalana d'Estudis Clàssics i l'Institut Català d'Arqueologia Clàssica, amb la colllaboració de la Facultat de Lletres de la Universitat Rovira i Virgili, pp. 133-151. Tarragona: Museu Nacional Arqueològic de Tarragona.

ESCALA, Óscar; MULET, Marta; COLET, Anna (2011): "El Tossal del Moro. Un jaciment visigoti ibèric a l'Urgell? Primers resultats". Urtx, 25, pp. 244-252.

ESCALA, Óscar; MOYA Andreu, TARTERA; Enric, VIDAL, Ares, ARMENTANO, Núria; NACIAROVA, Dominika, (2015): "La Fogonussa (Sant Martí de Riucorb). Ibers, romans i visigots a la comarca de
I'Urgell". Tribuna d'Arqueologia 2012-2013, pp. 245-261. Barcelona: Generalitat de Catalunya.

ESCALA, Óscar; COLET, Anna; MULET, Marta; MAZUQUE, Jordi; MARTíNEZ, Jordi (2018): "El jaciment ibèric i visigot del Tossal del Moro (Castellserà, l'Urgell). Darreres novetats". En Actes de les Primeres Jornades d'Arqueologia i Paleontologia de Ponent, pp. 202209. Lleida: Diputació de Lleida.

FOLCH, Cristian; GIBERT, Jordi; MARTÍN, Jairo; RODRIGO, Esther (2007): "L'ocupació de l'Alta edat mitjana del jaciment del Serrat dels Tres Hereus (Casserres, Berguedà)". En Actes del III Congrés d'Arqueologia Medieval i Moderna a Catalunya, pp. 753-757. Sabadell: Ajuntament de Sabadell, ACRAM.

FOLCH, Cristian; GIBERT, Jordi; MARTÍ, Ramon (2015): "Les explotacions rurals tardoantigues i altmedievals a la Catalunya Vella: una síntesi arqueològica". Estudis d'Història Agrària, 27, pp. 91-113.

FOLLIERI, M.; ROURE, J. M.; GIARDINI, M.; MAGRI, D.; NARCISI, B.; PANTALEÓN-CANO, J. e Yll, E. I. (2000): "Desertification trends in Spain and Italy based on pollen analysis". En BALABANIS, P.; PETER, D.; GHAZI, A. y TSOGAS, M. (eds.), International Conference on Mediterranean Desertification Research results and policy implications, pp. 33-44. European Commission.

FUENTES, Maribel (en prensa): "Intervenció arqueològica a l'antic hospital de clergues de Girona: l'evolució urbana de la riba del riu Galligants des de l'època romana fins a l'actualitat". Tribuna d'Arqueologia 2017-2018.

GARCIA, Moisés, MACIAS, Josep Maria; TEIXELL, Imma (1999): "Necròpoli de la vil.la dels Munts". Del romà al romànic. Història, art i cultura de la Tàrraconense mediterrània entre els segles IV i X, pp. 278-279. Barcelona: Enciclopèdia Catalana.

GUTIÉRREZ LLORET, Sonia (2007): "La islamización de Tudmir: balance y perspectivas". En SÉNAC, Philippe (éd.), Villes et campagnes de Tarraconaise et d'al-Andalus (Vle-Xle siècles): la transition, pp. 275-318. Paris: CNRS; Toulouse: Université de Toulouse-Le Mirail. https://doi.org/10.4000/books. pumi.25763

GUTIÉRREZ LLORET, Sonia (2013): «De Teodomiro a Tudmîr: los primeros tiempos desde la arqueología (S. VII-IX)》. En XXXIX Semana de Estudios Medievales: De Mahoma a Carlomagno. Los primeros tiempos (siglos VII-IX), pp. 229-283. Pamplona: Dpto. de Cultura, Turismo y Relaciones Institucionales.

HUNT, MarkA.; RAFEL, Núria; SORIANO, I. (2018): "The mine and the archaeological excavations". En RAFEL, Núria; HUNT ORTIZ, Mark A.; SORIANO, Ignacio; DELGADO-RAACK, Selina (eds.): Prehistoric copper mining in the north-east of the Iberian Peninsula: La Turquesa or Mas de les Moreres Mine (Cornudella de Montsant, Tarragona, Spain). Revista d'Arqueologia de Ponent, Número extra 3.

JÁRREGA, Ramón (2017): "Les ceràmiques romanes de la vil.la romana de Can Montagut (Marçà). Dades per a l'estudi del poblament rural romà al Priorat". Butlletí arqueològic, núm. 38-39 (20162017), pp. 81-139.

LING, J.; HJÄRTHNER-HOLDAR, E.; GRANDIN, L.; BILLSTRÖM, K.; PERSSON, P-O. (2013): "Moving metals or indigenous mining? Provenancing Scandinavian Bronze Age artefacts y lead isotopes and trace elements". Journal of Archaeological Science 40, pp. 291-304. https://doi.org/10.1016/j.jas.2012.05.040 
MARTÍ, Ramon (2013): "El palatium rural, una institución fiscal del siglo VIII". Lo que vino de Oriente. Horizontes, praxis y dimensión material de los sistemas de dominación fiscal en al-Andalus (siglos VII-IX), pp. 133-148. Oxford.

MARTÍNEZ ELCACHO, Albert (2014): "Pro crosis argenti". La plata a comtat de les Muntanyes de Prades i baronia d'Entença en època del comte Pere (1342-1358): regulació, gestió i rendiment de les mines de Falset (tesis doctoral). Universitat de Lleida. Lleida. Recuperado de: http://hdl.handle.net/10803/284994.

MENCHON, Joan (2012): "Necròpolis de l'antiguitat tardana i l'alta edat mitjana a les comarques del Camp de Tarragona, Conca de Barberà i Priorat". En MOLIST, Núria y RIPOLL, Gisela (eds.), Arqueologia funeraria al nord-est peninsular (segles VI-XII). Monografies d'Olèrdola 3.1, pp.125-154. Barcelona: Museu d'Arqueologia de Catalunya.

MENCHON, Joan (2013): "De l'Ager Tarraconensis a la Marca Extrema d'Alandalús. Algunes reflexions entorn al (des)poblament del Camp de Tarragona, Conca de Barberà i Priorat entre l'antiguitat tardana i la Conquesta feudal”. En PREVOSTI, Marta, LÓPEZ VILAR, Jordi y GUITART, Josep (eds.), Ager Tarraconensis 5. Paisatge, poblament, cultura material i historia, pp. 57-73. Tarragona: ICAC.

MERI, J. (ed.) (2006): Medieval Islamic civilization: an encyclopedia. Vol. I, pp. 816-818. Routledge, New Yok.

MOLIST, Núria; RIPOLL, Gisela (eds.) (2012): Arqueologia funeraria al nord-est peninsular (segles VI-XII). Monografies d'Olèrdola, 3.1 y 3.2. Barcelona: Museu d'Arqueologia de Catalunya-Olèrdola, 2 vols., $494 \mathrm{pp}$.

PALOL, Pere de (1989): El Bovalar (Seròs, Segrià): conjunt d'època paleocristiana i visigòtica. Lleida: Diputació de Lleida.

PÉREZ DÍAZ, Sebastián; LÓPEZ SÁEZ JoséAntonio (2018): “The plant context and pollen concentrates as a method of absolute dating at la Turquesa mine". En RAFEL, Núria, HUNT ORTIZ, MarkA., SORIANO, Ignacio y DELGADO-RAACK, Selina (eds.), Prehistoric copper mining in the north-east of the Iberian Peninsula: La Turquesa or Mas de les Moreres Mine (Cornudella de Montsant, Tarragona, Spain) Revista d’Arqueologia de Ponent, Número extra 3, pp. 41-47.

PIERA, Marc; MENCHON, Joan (2011): "El castell de Siurana (Cornudella de Montsant, el Priorat). Treballs arqueològics dels anys 2009-2010". Actes del IV Congrés d'Arqueologia Medieval i Moderna a Catalunya, pp. 867-878. Tarragona: Ajuntament de Tarragona, ACRAM.

RADEMAKERS, F. W.; NICOLAS N.; DE PUTTER, TH. y DEGRYSE, P. (2019): "Provenancing Central African copper croisettes: A first chemical and lead isotope characterisation of currencies in Central and Southern Africa". Journal of Archaeological Science 111 105010. https://doi.org/10.1016/j.jas.2019.105010

RAFEL, N.; ARMADA, X.L. (2010): "L'explotació minera en època romana al Baix Priorat (Tarragona): a propòsit del Plumbum Nigrum Oleastrense". Quaderns de Prehistória i Arqueologia de Castelló 28, pp. 247-261.

RAFEL, Núria; MONTERO, Ignacio; SORIANO, Ignacio; DELGADO-RAACK, Selina (2016): "L'activité minière préhistorique dans le Nord-Est de la péninsule Ibérique. Étude sur la Coveta de l'Heura et l'exploitation du cuivre à la Solana del Bepo (Tarragone, Espagne)". Bulletin de la Société préhistorique française Tome 113, numéro 1, pp. 95-129. https://doi.org/10.3406/bspf.2016.14720
RAFEL, Núria; HUNT ORTIZ, Mark A.; SORIANO, Ignacio; DELGADO-RAACK, Selina (eds.), (2018): Prehistoric copper mining in the north-east of the Iberian Peninsula: La Turquesa or Mas de les Moreres Mine (Cornudella de Montsant, Tarragona, Spain) Revista d'Arqueologia de Ponent, Número extra 3.

REIMER,P.J.;BARD, E.; BAYLISS, A.;BECK, J.W.; BLACKWELL; P.G., BRONK RAMSEY, C.; VAN DER PLICHT, J. (2013): "IntCal13 and MARINE13 radiocarbon age calibration curves $0-50000$ years cal BP". Radiocarbon, 55(4), pp. 1869-1887. https://doi.org/10.2458/azu_js_rc.55.16947

RIERA, Santiago (2003): "Evolució vegetal al sector de Vilanova-Cubelles (Garraf) en els darrers 3000 anys: processos naturals i transformacions antròpiques d'una plana litoral mediterrània". En GUITART, Josep, PALET, Josep Maria y PREVOSTI, Marta (eds.), Territoris antics a la Mediterrània i a la Cossetània oriental. Actes del Simposi Internacional d'Arqueologia del Baix Penedès, pp. 303-312. Barcelona: Generalitat de Catalunya.

RIERA, Santiago; MIRAS, Yannick; GIRALT, Santiago; SERVERA, Gabriel (2010): "Evolució del paisatge vegetal al Camp de Tarragona: estudi pollínic de la seqüència sedimentològica procedent de la Sèquia Major (La Pineda, Vila-seca)». En PREVOSTI, Marta y GUITART, Josep (eds.), Ager Tarraconensis 1. Aspectes històrics i marc natural, pp.163-173. Tarragona: ICAC.

RIPOLL, Gisela; ARCE y Javier (2001): "Transformación y final de las villae en occidente (siglos IV-VIII): problemas y perspectivas". Arqueología Medieval, 8, pp. 21-54. https://doi.org/10.17561/ aytm.v8i0.1672

RIPOLL, Gisela; CARRERO, Eduardo; RICO, Daniel, MOLIST; Núria, CENTELLES, Àngela G.; BENSEY, Josep; TUSET, Francesc (2017): "Sancti Cirici de Colera / Sant Quirze de Colera (Alt Ampordà, Girona). Estudio preliminar del conjunto monástico, siglos VIII al XVI." Hortus Artium Mediaevalium, 23(2), pp. 602-628. https://doi. org/10.1484/J.HAM.5.113750

ROVIRA, Jordi; BATISTA, Ricardy MOLIST, Núria (1991): "El conjunt monumental d' Olèrdola: les darreres campanyes d'excavacions (1983-1989)". Tribuna d'Arqueologia 1989-1990, pp. 87-100. BarceIona: Generalitat de Catalunya.

ROVIRA, Salvador; MONTERO, Ignacio; CONSUEGRA, Susana (1997): La primeras etapas metalúrgicas en la Península Ibérica. I Análisis de materiales. Madrid: Instituto Universitario Ortega y Gasset.

ROVIRA LLORENS, S.; MONTERO RUIZ, I. (2018): "Proyecto de arqueometalurgia de la Península Ibérica (1982-2017)”. Trabajos de Prehistoria, 75 (2), pp. 223-247. https://doi.org/10.3989/ tp.2018.12213

SALES, JORDINA (2014): "El Bovalar (Serós, Lleida). ¿Un monasterio productor de pergamino en la Hispania visigoda?". RaCr 90, pp. 423-464.

SANCHO i PLANAS, Marta (2018): "Recursos alimentaris en el monestir d'època visigoda de Santa Cecília de Els Altimiris (Sant Esteve de la Sarga-Pallars Jussà). Primeres aportacions". Revista d’Arqueologia de Ponent, núm. 28, pp. 63-80. https://doi. org/10.21001/rap.2018.28.3

SANCHO i PLANAS, Marta; ALEGRÍA TEJEDOR, Walter (2017): "Propuesta de contextualización del yacimiento tardoantíguo y altomedieval de Els Altimiris (Prepirineo Leridano) siglos V-IX". Archeologia Medievale, XLIV, pp. 155-170. 
SOLANES, Eva; ALÒS Carme (2003): "Interpretació de l'aixovar de la necròpolis visigòtica de Palous (Camarasa, la Noguera): apunts sobre l'adobatge de pells a l'antiguitat tardana". Revista d'Arqueologia de Ponent 13, pp. 345-350.

SORIANO, Ignacio; HUNT ORTIZ, Mark A. (2018): "Minería y metalurgia prehistóricas en el Priorat y zonas limítrofes (provincia de Tarragona). Estado de la cuestión". Revista d'Arqueologia de Ponent, núm. 28, pp. 329-340. https://doi.org/10.21001/rap.2018.28.18

VALLVÉ, J. (1980): "La industria en Al-Andalus". Al-Qantara I, pp. 209-241.

VALLVÉ, J. (1996): "La minería en Al-Andalus". Actas de las I Jornadas sobre minería y tecnología en la Edad Media Peninsular, pp. 56-64.
VILASECA, Salvador (1930): "Enterraments d'època romana a Reus i el Molar". Revista del Centre de Lectura de Reus, III època, núm. 212, pp. 324-327.

VILASECA, Salvador (1934): "Les coves d'Arbolí (Camp de Tarragona)". Butlletí Arqueològic, època III, núm. 49, pp. 373-388.

VILASECA, Salvador (1952): "La coveta de l'Heura, de Ulldemolins (provincia de Tarragona)". Ampurias XIV: pp. 121-135.

ZOZAYA, Juan (2010): "La línea de fortificaciones andalusíes del Duero Oriental". Actas del Coloquio Internacional Patrimonio Cultural y Territorio en el Valle del Duero. Zamora, 28, 29 y 30 de marzo de 2007, pp. 235-255. Salamanca: Fundación Rey Alfonso Henriques. 